\title{
Genes and screens
}

\section{Biotechnology-related products take centre stage.}

Brilliant

Stratagene

www.stratagene.com

Mix with these

Two master mixes for quantitative PCR are available from Stratagene, with or without SYBR Green 1 dye. The pre-mixed formulations include Taq DNA polymerase with hotstart capability, GAUC mixture for optional dUTP/UNG carryover contamination control, and optimized buffer. The mixes have been formulated to work on most quantitative PCR platforms.

\section{Fastblot B64}

Biometra www.biometra.de

Large-capacity gel blotting

Biometra's semi-dryblotting family has been extended to include Fastblot B64. This proteomics blotter, which has a blotting area of $23.5 \times 38.5 \mathrm{~cm}$, is designed for blotting large, two-dimensional gels and sequencing gels, or for simultaneous transfer of multiple gels. Both continuous and discontinuous buffer systems with pH 3-9 may be used, as well as SDS. Proteins and nucleic acids are typically transferred from gels in 10-30 minutes. A spring-supported anode allows the use of thicker gels (up to $5 \mathrm{~mm}$ ) and gel stacks, and eliminates the need for a special frame for blotting of agarose gels.

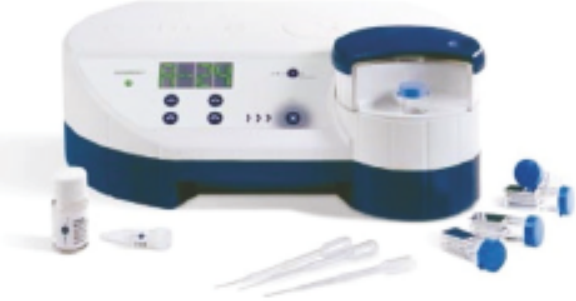

Nucle ofector makes gene transfer safe and easy.

MICROMAX

PerkinElmer Life Sciences

www.perkinelmer.com/lifesciences

Microarrays with Incyte

PerkinElmer's MICROMAX Human cDNA Microarray System II-TSA incorporates gene content from Incyte Genomics. This new microarray features sequence-verified Human Drug Target Genes mined from Incyte Genomics' LifeSeq Gold database, combined with TSA amplification technology. The system is equipped with two slides containing some 5,000 genes per slide, two practice/control microarrays, six coverslips, two plastic templates and a TSA labelling and detection system. Also available is MICROMAX Microarray: Human CDNA II, which includes two slides pre-spotted with the same Incyte Genomics content, but without practice microarrays or the TSA system.
Nucleofector

Amaxa

www.amaxa.com Safe, non-viral gene transfer

Human mesenchymal stem cells (HMSC) are adult stem cells with the ability to differentiate into a variety of tissue-specific cell types. Amaxa's HMSC Nucleofector kit uses a safe, non-viral gene transfer method for primary cells and gene lines that are difficult to transfect. By transferring DNA directly into the cellnucleus, it allows cells with limited ability to divide, such as primary cells, to be madeaccessible for gene transfer.

\section{Tissue microarrays}

InnoGenex

www.innogenex.com Rapid screening of in situ gene expression

InnoGenex tissue microarrays are available from diseased and normal human tissues, and normal mouse and rat tissues. They are suitable for rapid screening of in situ differential expression of genes, identifying novel antibody markers and animal model studies. Arrays contain $20-50$ or $100-200$ elements per slide. All donor tissue blocks and final tissue arrays are validated for use in immunohistochemistry and in situ hybridization assays.

These notes are compiled in the Nature office from information provided by the manufacturers 